\title{
New high-risk molecular subtypes of DLBCL identified
}

So-called double-hit diffuse large B cell lymphomas (DLBCLs) with both $M Y C$ and BCL2 rearrangements are known to hold an unfavourable prognosis. Now, two studies reveal new subsets of tumours with overlapping high-risk phenotypes.

In one of the studies, David Scott and colleagues defined a 104-gene expression signature (DHITsig) that distinguished double-hit DLBCLs from other germinal centre $B$ cell-like (GCB)-DLBCLs, which are typically associated with a favourable prognosis. However, only $52 \%$ of patients with DHITsig-positive disease were found to have $M Y C$ and $B C L 2$ rearrangements. Importantly, in retrospective analyses of patients treated with R-CHOP immunochemotherapy, positivity for the DHITsig was associated with inferior outcomes, irrespective of $M Y C / B C L 2$ status. Using a different gene expression signature previously observed in Burkitt lymphoma (a disease related to, but generally more aggressive than, DLBCL), another group of investigators led by David Westhead similarly identified a subset of poor prognosis 'molecular high grade' (MHG) DLBCLs, 50\% of which were defined cytogenetically as double-hit DLBCLs. These findings essentially double the number of patients with high-risk, double-hit phenotype GCB-DLBCL, such that they account for $\sim 15 \%$ of all patients with DLBCL.

"In clinical practice, double-hit DLBCLs are increasingly treated differently to other GCB-DLBCLs, most often using more intensive chemotherapy, and we suggest that this practice might usefully be extended to the whole MHG group," says Westhead. By contrast, "after identifying and removing patients with DHITsig-positive tumours, the GCB-DLBCL population now has an outstanding prognosis

(5-year disease-specific survival of $\sim 90 \%$ ), obviating the need for treatment escalation in this group," adds Scott.

Regarding the way forwards, "the first step will be to retrospectively apply this new classification system to completed clinical trials in order to determine whether existing treatment regimens will be useful," states Scott. Additionally, "we are starting to design prospective trials in the high-risk group, initially with more intensive chemotherapy, but ultimately with agents targeting the particularities of the biology," Westhead concludes. Importantly, Scott and Westhead are comparing their findings with the aim of forming a consensus definition for prospective clinical use.

David Killock number of patients with high-risk, double-hit phenotype...

\section{HAEMATOLOGICAL CANCER}

\section{MRD to help assess response in CLL}

Standard-of-care therapies for patients with relapsed and/or refractory (R/R) chronic lymphocytic leukaemia (CLL) are shifting from being chemoimmunotherapy based to incorporating targeted agents such as venetoclax, an inhibitor of apoptotic regulator BCL-2. Now, updated results of the phase III MURANO trial confirm a progression-free survival (PFS) benefit with venetoclax in CLL.

Patients with R/R CLL were randomly assigned to receive either 6 cycles of venetoclax plus rituximab and then venetoclax monotherapy for an additional 18 months (fixedduration regimen; $n=194$ ) or 6 cycles of bendamustine plus rituximab (standard regimen; $n=195$ ). In an earlier analysis (median follow-up duration of 23.8 months), PFS was superior with the fixed-duration regimen over standard therapy. "At the time of analysis, most patients were still receiving venetoclax. The question was whether survival curves would remain favourable after treatment cessation," explains lead investigator Arnon Kater.

For the 130 patients (65\%) who completed treatment with venetoclax, the median duration of treatment was 24.4 months. At a median follow-up duration of 36.0 months, PFS remained longer with the fixed-duration regimen than with standard treatment: not reached versus 17.0 months (HR $0.16 ; P<0.001)$. The estimated 3 -year overall survival was $87.9 \%$ versus $79.5 \%$. In the earlier publication, the reported frequencies of grade 3-4 adverse events were $82.0 \%$ with the undetectable or low levels of MRD ... [are] a desirable goal of CLL therapies leukocytes) was substantially higher with the fixed-duration than with fixed-duration regimen and $70.2 \%$ with the standard regimen.

A secondary end point of the study was the assessment of minimal residual disease (MRD) in peripheral blood 2-3 months after the end of combination therapy. The rate of undetectable MRD ( $<1$ CLL cell per $10^{4}$ the standard regimen $(82.5 \%$ versus $23.1 \%)$. In either treatment arm, PFS durations were longer for patients with undetectable MRD than for those with detectable MRD. "Our results indicate that undetectable or low levels of MRD (1 CLL cell per $10^{2}-10^{4}$ leukocytes) are meaningful end points and a desirable goal of CLL therapies," summarizes Kater.

On the importance of these results, Kater adds "Findings on MRD provide data that will be implemented in new trials aiming to investigate limiting the length of fixed-duration therapy. This aspect is highly desired by both patients and health authorities owing to the long-term toxicities (including financial toxicities) associated with maintenance therapy."

Diana Romero
ORIGINAL ARTICLE Kater A. P.et al. Fixed duration of venetoclax-rituximab in relapsed/ refractory chronic lymphocytic leukemia eradicates minimal residual disease and prolongs survival: post-treatment follow-up of the MURANO phase III study.J. Clin. Oncol. https://doi.org/10.1200/JCO.18.01580 (2018) FURTHER READING Seymour, J. F. et al. Venetoclax-rituximab in relapsed or refractory chronic lymphocytic leukemia. N. Engl.J. Med. 378 $1107-1120(2018)$ 